\title{
Photon beam relative dose validation of the DPM Monte Carlo code in lung-equivalent media
}

\author{
Indrin J. Chetty, ${ }^{\text {a) }}$ Paule M. Charland, Neelam Tyagi, Daniel L. McShan, \\ and Benedick A. Fraass \\ The University of Michigan, Department of Radiation Oncology, Ann Arbor, Michigan 48109-0010 \\ Alex F. Bielajew \\ The University of Michigan, Department of Nuclear Engineering, Ann Arbor, Michigan 48109-2104
}

(Received 8 November 2002; accepted for publication 2 January 2003; published 25 March 2003)

\begin{abstract}
Validation experiments have been conducted using 6 and $15 \mathrm{MV}$ photons in inhomogeneous (water/ lung/water) media to benchmark the accuracy of the DPM Monte Carlo code for photon beam dose calculations. Small field sizes (down to $2 \times 2 \mathrm{~cm}^{2}$ ) and low-density media were chosen for this investigation because the intent was to test the DPM code under conditions where lateral electronic disequilibrium effects are emphasized. The treatment head components of a Varian 21EX linear accelerator, including the jaws (defining field sizes of $2 \times 2,3 \times 3$ and $10 \times 10 \mathrm{~cm}^{2}$ ), were simulated using the BEAMnrc code. The phase space files were integrated within the DPM code system, and central axis depth dose and profile calculations were compared against diode measurements in a homogeneous water phantom in order to validate the phase space. Results of the homogeneous phantom study indicated that the relative differences between DPM calculations and measurements were within $\pm 1 \%$ (based on the rms deviation) for the depth dose curves; relative profile dose differences were on average within $\pm 1 \% / 1 \mathrm{~mm}$. Depth dose and profile measurements were carried out using an ion-chamber and film, within an inhomogeneous phantom consisting of a $6 \mathrm{~cm}$ slab of lung-equivalent material embedded within solid water. For the inhomogeneous phantom experiment, DPM depth dose calculations were within $\pm 1 \%$ (based on the rms deviation) of measurements; relative profile differences at depths within and beyond the lung were, on average, within $\pm 2 \%$ in the inner and outer beam regions, and within 1-2 $\mathrm{mm}$ distance-to-agreement within the penumbral region. Relative point differences on the order of $2-3 \%$ were within the estimated experimental uncertainties. This work demonstrates that the DPM Monte Carlo code is capable of accurate photon beam dose calculations in situations where lateral electron disequilibrium effects are pronounced. (C) 2003 American Association of Physicists in Medicine.
\end{abstract}

[DOI: $10.1118 / 1.1555671]$

Key words: DPM Monte Carlo code, phase space, BEAMnrc, lateral electronic disequilibrium, ion chamber measurements

\section{INTRODUCTION}

Experimental validation of dose calculation models is an important step before the implementation of these algorithms in a clinical setting. Suggested dose accuracy for commissioning of treatment planning systems is typically $2 \% / 2 \mathrm{~mm}$ in the high dose and penumbral regions, respectively, in homogeneous phantoms. These criteria are increased to $4 \% / 4 \mathrm{~mm}$ in the presence of 3-D inhomogeneities, ${ }^{1}$ where conventional dose algorithms do not offer explicit electron transport that is usually required to accurately characterize the perturbative effect of the inhomogeneity. The emergence of model-based dose calculation techniques, such as the convolution/ superposition and the Monte Carlo methods, provide a more physics-based approach that has been found by many investigators to be more accurate than correction-based methods for calculating the dose in inhomogeneous media. ${ }^{2-5}$ In particular, the Monte Carlo method is currently the only method that explicitly transports photons and electrons within a material and is therefore likely to provide more accurate results at material interfaces and within inhomogeneities. To date, researchers have conducted a wide range of experiments in both homogeneous and inhomogeneous geometries to validate user-specific Monte Carlo codes developed for clinical treatment planning. ${ }^{6-21}$

As physically realistic approaches become more practical for dose calculations, it becomes critical that these models be adequately validated against measurements. The increase in sophistication of dose algorithms also means that experimental validation should include complex geometries that aim to test the limits of the codes used. The focus of this work was to conduct experimental validation of the DPM (Dose Planning Method) Monte Carlo code for photon beam calculations in situations where lateral electron disequilibrium effects are emphasized, as observed, for example, when using small field sizes $\left(2 \times 2 \mathrm{~cm}^{2}\right)$, low-density media (lungequivalent slabs) and high energies (15 MV photons). It was expected that such measurements would provide a stringent assessment of the transport physics employed within the DPM code.

Details of the electron/photon transport model used in 
DPM are provided in the paper by Sempau et al. ${ }^{10}$ however, a few general comments on the transport physics are in order here. Electron transport within DPM uses a condensed history model that is based on a Class II "mixed" transport scheme for energy losses, with analog transport for large energy transfers, and the continuous slowing down approximation (CSDA) used for small energy losses. ${ }^{10}$ DPM employs several features which make it optimal for radiotherapy class dose calculations. These include (a) the use of a step size independent multiple scattering theory based on the KawrakowBielajew formalism, ${ }^{10}$ (b) the use of a "random hinge" scheme for transporting charged particles from point-to-point in the medium, originally developed in the PENELOPE code $^{22}$ but modified within DPM to provide a basis for simulating scattering across material boundaries, (c) the use of large electron steps which affords the ability to traverse many voxels before sampling a multiple scattering angle, and (d) the use of Woodcock tracking (delta-scattering) to reduce the overheads associated with transporting photons across boundaries.

In this paper we present an investigation of the accuracy of DPM calculations versus measurements for photon beams incident upon a low-density composite water equivalent-lung equivalent phantom, for field sizes of $2 \times 2,3 \times 3$, and 10 $\times 10 \mathrm{~cm}^{2}$. DPM calculations have initially been benchmarked in homogeneous geometries to validate the accuracy of the phase space simulation of the accelerator treatment head and ensure accurate modeling of the source. It should be noted that we have not included field sizes larger than 10 $\times 10 \mathrm{~cm}^{2}$ in this study because the intent of this work was to investigate transport accuracy issues, which are best evaluated at small field sizes in low-density media. In describing the details of this work, the following topics will be addressed: Monte Carlo simulation of the linear accelerator treatment head using BEAMnrc, the experimental setup and measurement details, DPM simulation results and comparisons with measurements in both homogeneous and inhomogeneous phantoms.

\section{SIMULATION OF THE LINEAR ACCELERATOR TREATMENT HEAD}

A detailed phase space simulation of the components of a Varian 21EX linear accelerator (Varian Associates, Palo Alto, CA) was conducted using the usercode BEAMnrc ${ }^{23}$ (based on EGSnrc $^{24}$ transport physics). The 21EX linac is an isocentric machine that produces two photon beam energies ( 6 and 15 MV) and five electron beams, from 6 to $20 \mathrm{MeV}$. BEAMnrc includes a comprehensive simulation geometry package that provides several component modules (CMs) with which to model various structures within the accelerator treatment head. ${ }^{23}$ The specific CMs used for this study were the following: SLAB for the vacuum window, CONSTAK for the target and target housing, CONS3R for the primary collimator, FLATFILT for the 6 and 15 MV flattening filters, CHAMBER for the transmission chamber, MIRROR for the mirror, and JAWS for the secondary ( $x$ and $y$ ) collimators. The multi-leaf collimator was not included in this simulation

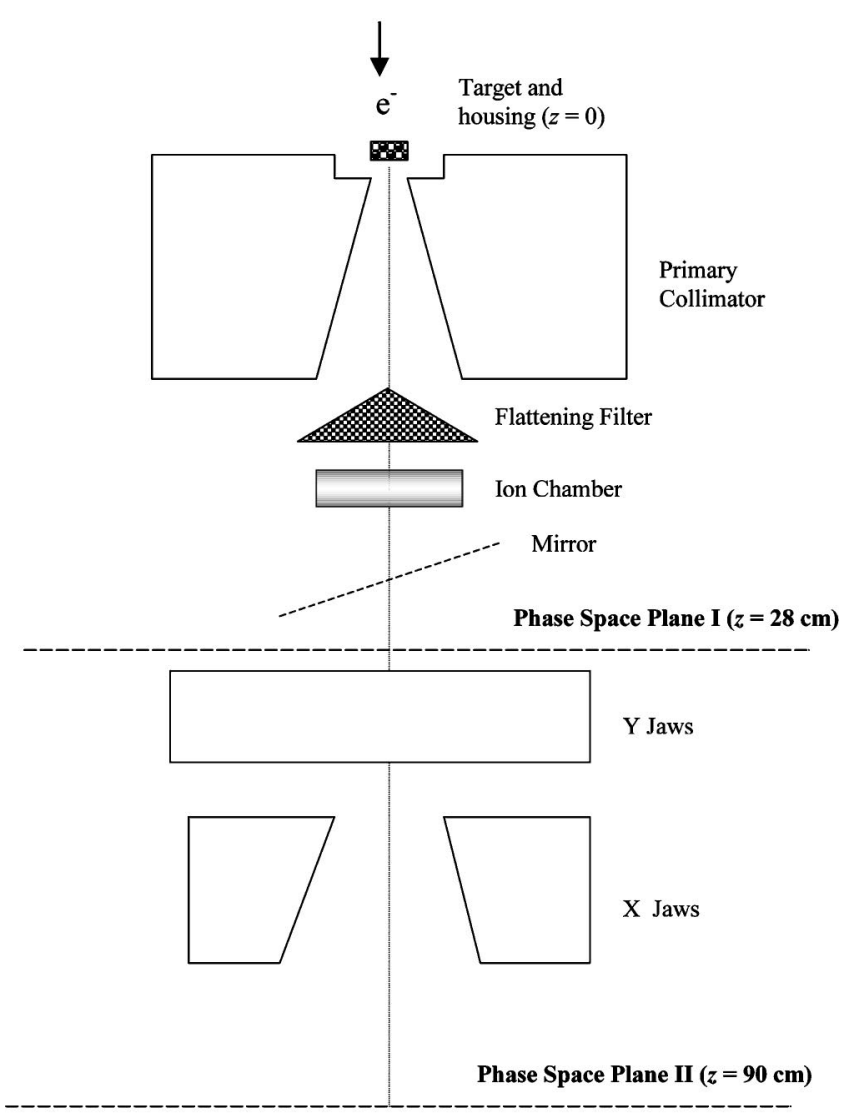

FIG. 1. BEAMnrc simulation geometry illustrating the treatment head components of the linear accelerator. The phase-space scored at plane I included only the patient-independent structures, while that scored at plane II included only the field-defining jaws.

and was retracted for all measurements performed in this study. Figure 1 illustrates the various components in the accelerator treatment head that were included in the simulation. In order to increase the efficiency of the simulation, a twostep process was utilized. In the first step, the phase space was tallied at a scoring plane (see phase space plane I, Fig. 1) located $28 \mathrm{~cm}$ downstream from the target and perpendicular to the beam central axis (CAX). This simulation included the "patient-independent" structures: the target, primary collimator, flattening filter, transmission chamber, and mirror. Specifically, the following parameters were scored for each history: $x, y, u, v$, energy, latch, and weight. The second step of the simulation process involved transport through the "patient-dependent" structures (the $x$ and $y$ secondary jaws). The jaws were set to define field sizes of $2 \times 2,3 \times 3$, and $10 \times 10 \mathrm{~cm}^{2}$ at the isocenter, and the phase space was scored (for each field size independently) at a plane $90 \mathrm{~cm}$ downstream from the target (see phase space plane II, Fig. 1). Note that the source particle parameters for the field-sizedependent simulation were contained in the phase space file acquired at plane I. All BEAMnrc phase space calculations in this work used default EGSnrc physics parameters.

The field-size-dependent phase space files were ported to the DPM code system for dose calculations within the phantom. A trial and error method, similar to that used by other 
investigators, ${ }^{11,17,23,25}$ was used to "calibrate" the incident electron-on-target energy for the initial phase space simulation. This involved adjusting the incident electron-on-target energy in the initial phase space simulations to provide the best fit between DPM calculations and measurements for central axis depth dose and profiles for a $10 \times 10 \mathrm{~cm}^{2}$ field size, $90 \mathrm{~cm} \mathrm{SSD}$, in water. For this study, the beam of electronson-target was modeled as a mono-energetic, parallel source of electrons with no angular spread. The "calibrated" electron-on-target energies were found to be 6.25 and 15.3 $\mathrm{MeV}$ for 6 and $15 \mathrm{MV}$ photons, respectively.

\section{PHANTOM MEASUREMENTS}

\section{A. Homogeneous phantom measurements}

Central axis depth and profile doses were measured in the Scanditronix/Wellhöfer water scanning system using an SFD stereotactic diode (Scanditronix, Uppsala, Sweden) with a 2 $\mathrm{mm}$ active area diameter, and a $0.06 \mathrm{~mm}$ active volume thickness. The dimensions of this water phantom are 40 $\times 40 \times 38 \mathrm{~cm}^{3}$. Measurements were conducted for 6 and 15 MV photons incident at $90 \mathrm{~cm} \mathrm{SSD,} \mathrm{for} \mathrm{field} \mathrm{sizes} \mathrm{of} 2$ $\times 2,3 \times 3$ and $10 \times 10 \mathrm{~cm}^{2}$, defined at the isocenter. Profiles were measured at depths ranging from $d_{\max }(1.5 \mathrm{~cm}$ for 6 $\mathrm{MV}$ and $3.0 \mathrm{~cm}$ for $15 \mathrm{MV}$ ) to $30 \mathrm{~cm}$. The photon diode was chosen for these measurements because of its superior spatial resolution which is necessary for accurately measuring small field profiles. Diodes have been used by other investigators ${ }^{7}$ for small field measurements because of their enhanced spatial resolution.

\section{B. Inhomogeneous phantom measurements}

The inhomogeneous phantom consisted of slabs of solid water (Gammex RMI, Middleton WI) with dimensions of $30 \times 30 \mathrm{~cm}^{2}$ and varying thicknesses; the solid water material, from depths of $4 \mathrm{~cm}$ to $10 \mathrm{~cm}$, was replaced with a $6 \mathrm{~cm}$ thick lung-equivalent full slab phantom of density $0.3 \mathrm{~g} / \mathrm{cm}^{3}$ (Gammex RMI, Middleton WI). It should be noted that the 6 $\mathrm{cm}$ lung-equivalent slab was constructed by re-arranging smaller pieces of lung-equivalent material of varying dimensions (varying in width, length, and thickness) to form the slab. The experimental setup for measurements within the inhomogeneous phantom is illustrated in Fig. 2. For the central axis depth dose measurements, an IC-10 (Scanditronix, Uppsala, Sweden) cylindrical ion chamber ionization chamber, with an air cavity volume of $0.13 \mathrm{~cm}^{3}$ and a $3 \mathrm{~mm}$ inner radius, was inserted at the following depths within the phantom: 1.0, $d_{\max }, 2.0,3.0,5.0,7.0,9.0,11.0,13.0,15.0$, and $20.0 \mathrm{~cm}$. Charge (in $n \mathrm{C}$ ) was collected with a PRM Model SH-1 (Precision Radiation Measurements, Tennessee) electrometer operated at a $300 \mathrm{~V}$ bias. A set of 3 readings, with exposures of $100 \mathrm{MU}$ per reading (corresponding to the calibration dose of $80 \mathrm{cGy}$ at $10 \mathrm{~cm}$ depth, $90 \mathrm{~cm} \mathrm{SSD,} 10$ $\times 10 \mathrm{~cm}^{2}$ in water), was acquired at each point. The effective point of measurement for the cylindrical ion chamber was taken into consideration by shifting the measured depth dose curve $1.8 \mathrm{~mm}$ for the $6 \mathrm{MV}$ beam and $2.0 \mathrm{~mm}$ for the $15 \mathrm{MV}$

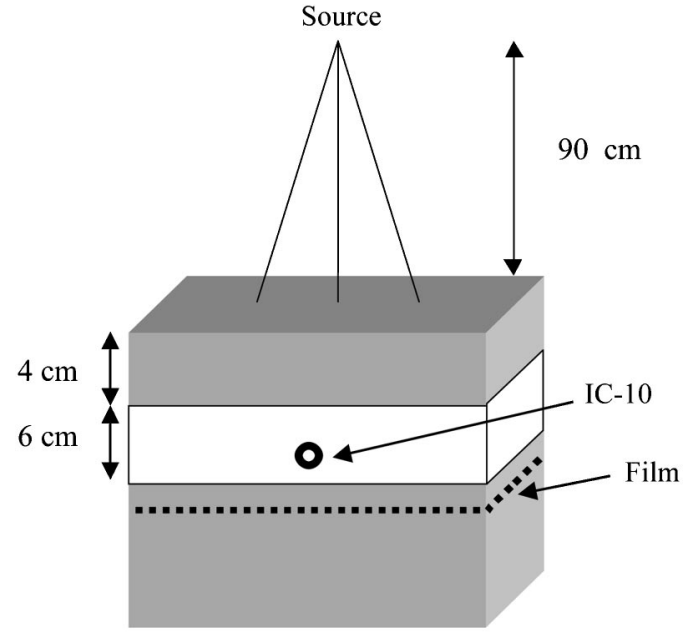

FIG. 2. Experimental geometry for the inhomogeneity measurements. The lung-equivalent slab extends from $4-10 \mathrm{~cm}$ within the solid water. An IC-10 ion chamber was inserted at various depths, within the solid water and lung, to measure the central axis depth dose. Film was inserted between slabs of solid water to measure profile doses.

beam, as recommended in the Scanditronix/Wellhöfer scanning system manual (Scanditronix, Uppsala, Sweden) and also used by others. ${ }^{7}$

Profiles along the central $x$-axis were measured with the IC-10 chamber at depths of $8 \mathrm{~cm}$ and $12 \mathrm{~cm}$. This was accomplished by translating and repositioning the phantom so as to increment the chamber off-axis, by $0.5 \mathrm{~cm}$ in the high dose region of the profile and by $0.2 \mathrm{~cm}$ in the profile penumbral (high dose gradient) region. A graph paper with millimeter spacing was used to conduct the alignment and translation during this particular experiment. Profiles within the inhomogeneous phantom were also measured using Kodak Ready-Pack Extended Dose Range (EDR) film (Eastman Kodak Co., Rochester, NY). A recent study has shown that Kodak EDR film is dosimetrically comparable to the widely used Kodak XV film over a much larger range of doses. ${ }^{26}$ In this experiment, films were sandwiched in the inhomogeneous phantom at depths of $8 \mathrm{~cm}$ and $12 \mathrm{~cm}$. Each film pack was pin-pricked to avoid unwanted air in the envelope. Irradiated films were processed using a Kodak X_Omat-3000 RA, automatic film processor with a 90 second processing time. Processed films were converted to digitized images using the Lumiscan Model 100 (Lumisys, Sunnyvale, CA) laser digitizer with a $0.42 \mathrm{~mm}$ spot size and $0.45 \mathrm{~mm}$ pixel size. Profile images were analyzed with the Fuji film analysis software, ScienceLab 98-Image Gauge (Fuji Photo film Co, Ltd., Itasca, IL). The conversion from optical density to dose was carried out using an H\&D or sensitometric curve for Kodak EDR film. Sensitometric curves were generated in solid water for 6 and $15 \mathrm{MV}$ photons at a depth of $5 \mathrm{~cm}$ for a $10 \times 10 \mathrm{~cm}^{2}$ at $90 \mathrm{~cm} \mathrm{SSD}$, by exposing films (perpendicularly to the central axis) to varying doses from 0 to $400 \mathrm{cGy}$ (in 12 equal dose increments), within the linear region of the sensitometric curve for Kodak EDR film. Optical densities for films irradiated for sensitometric analysis were read out using a Digital Densitometer II (Sun Nuclear Corporation, 
Melbourne, FL) optical densitometer. Although the sensitometric curves were measured at a depth different than that of the profiles, the variation in sensitometric response with depth for the field sizes studied in this work was found to be negligible. ${ }^{26}$

\section{Uncertainty estimates}

For the diode depth dose and profile measurements in water, the uncertainty was estimated to be less than $0.5 \mathrm{~mm}$ in depth, relative to the surface. Positioning of the IC-10 ion chamber at a given depth was estimated to be within $1 \mathrm{~mm}$, relative to the center of the chamber. Precision of the IC-10 chamber based on the reproducibility of at least three readings per point was found to be within $\pm 0.3 \%(1 \sigma)$ for all points measured. Measurements were taken at the beginning of the session and repeated a few hours later, at the end, to estimate drifts in the output of the accelerator. These differences were within $\pm 0.3 \%$. The uncertainty associated with translation and alignment of the ion chamber with the crosshairs during profile measurements in the inhomogeneous phantom was estimated to be within $1 \mathrm{~mm}(2 \sigma)$. For film measurements, uncertainties were estimated by identically irradiating and processing a set of eight films; the variation in optical density was found to be approximately $\pm 2 \%(1 \sigma)$.

\section{MONTE CARLO CALCULATIONS}

\section{A. Phase space source description}

DPM calculations were conducted within a voxelized phantom using, for the input source description, the phase space files (at phase space plane II, Fig. 1) generated with BEAMnrc for 6 and $15 \mathrm{MV}$ photons at $90 \mathrm{~cm} \mathrm{SSD} \mathrm{in} \mathrm{air,} \mathrm{for}$ $2 \times 2,3 \times 3$, and $10 \times 10 \mathrm{~cm}^{2}$ field sizes as specified at the isocenter. Each record in the phase-space files contained the following parameters for each particle, in binary format: $x$, $y, u, v$, energy, latch, and weight. $X$ and $y$ represent the particle's position in the phase space plane (at a fixed $z$-location), $u$ and $v$, the particle's direction cosine vectors along the $x$ and $y$ axes, respectively. The latch variable, in addition to storing the particle's creation/interaction history (dependent upon the latch type), also contains a bit to distinguish whether the particle is an electron or a photon. ${ }^{23} \mathrm{~A}$ sub-routine was written to read the phase space parameters directly within the DPM code. Included within this routine is a calculation to determine $w$ (the direction cosine vector along the $z$-axis) for each particle, based upon the method employed in DOSXYZnrc ${ }^{16}$-this calculation uses the identity $u^{2}+v^{2}+w^{2}=1$.

\section{B. Monte Carlo uncertainty estimates}

Both the DPM and BEAMnrc Monte Carlo codes utilize the history-by-history method for estimating the standard deviation, $S_{\bar{x}}$. This method has been described by Sempau et al., ${ }^{21}$ Briesmeister, ${ }^{27}$ Walters et al.,${ }^{28}$ and Andreo. ${ }^{2} S_{\bar{x}}$ is calculated using the equation:

$$
s_{\bar{x}}=\sqrt{\frac{1}{N-1}\left(\frac{\sum_{i=1}^{N} X_{i}^{2}}{N}-\left(\frac{\sum_{i=1}^{N} X_{i}}{N}\right)^{2}\right)},
$$

where $N$ is the number of histories and $X_{i}$ the quantity of interest (such as dose) scored in independent history $i$. The advantages of the history-by-history statistical estimator over the batch method are detailed in the paper by Walters et al. ${ }^{28}$

BEAMnrc calculations for the patient-independent structures (i.e., phase space acquired at plane I in Fig. 1) contained approximately 100 million histories in the phase space files for both 6 and $15 \mathrm{MV}$ photons; the $1 \sigma$ statistics in photon fluence was, on average, less than $0.5 \%$ for these runs. For calculations including the secondary jaws (i.e., phase space acquired at plane II in Fig. 1), the number of phase space records ranged from 2 million for the 2 $\times 2 \mathrm{~cm}^{2}$ field to 11 million for the $10 \times 10 \mathrm{~cm}^{2}$ field, resulting in average statistics ( $1 \sigma$ in photon fluence) ranging from $0.5-1.0 \%$. Sempau et al. ${ }^{21}$ have coined the term "latent uncertainty" to describe the uncertainty due to statistical fluctuations in the phase space; this is distinct from the uncertainty due to the random nature of the transport in phantom. As pointed out by Walters et al. ${ }^{28}$ the statistical uncertainty in calculated dose will approach the finite, latent uncertainty associated with the phase space, regardless of the number of times the phase space is sampled. In this work, the latent uncertainty was estimated by summing the uncertainties from phase space planes I and II in quadrature. In order to estimate the total uncertainty in the calculated dose in phantom, we have quadrature summed the uncertainties from the DPM phantom calculations (due to the random fluctuations in phantom) with the inherent (latent) uncertainty of the phase space (see Table I). These uncertainties fall roughly in the range from $0.5-1.5 \%$ (see Table I), for all points in this study.

\section{DPM physics and scoring parameters}

Calculations using the DPM Monte Carlo code were performed using a simulated cubic water phantom (with a side of $40 \mathrm{~cm}$ ) for the homogenous geometry and a composite water-lung-water-equivalent phantom for the heterogeneous calculations. A scoring voxel with dimensions $2 \times 2$ $\times 2 \mathrm{~mm}^{3}$ was used for most calculations; this was reduced to $1 \mathrm{~mm}$ in the scoring axis to obtain finer resolution for the smaller field $\left(2 \times 2\right.$, and $\left.3 \times 3 \mathrm{~cm}^{2}\right)$ profiles. All calculations were conducted in water for a single, AP beam, perpendicularly incident on the phantom at $90 \mathrm{~cm} \mathrm{SSD}$, to mimic the measurement geometry. No differentiation was made between water and solid water in the input files for calculations in the lung-equivalent phantom; studies ${ }^{26,29}$ have shown that relative depth dose differences between solid water and water are small $(<1.0 \%)$, confirming that the comparison between measurements in water and Monte Carlo calculation solid water is unbiased by minor differences in the electron densities between these two materials. The lung-equivalent material was modeled as a slab with a uniform physical density of $0.3 \mathrm{~g} / \mathrm{cm}^{3}$ and an atomic composition specified by the 
TABLE I. Quantitative analysis of the DPM calculated and measured depth dose curves illustrated in Figs. 4 and 7. Specifically shown are the rms deviations and the maximum point differences, the DPM $(1 \sigma)$ uncertainty range and the total $(1 \sigma) \mathrm{MC}$ uncertainty range. The total $\mathrm{MC}$ uncertainty was estimated by summing the latent phase space and DPM uncertainties in quadrature.

\begin{tabular}{|c|c|c|c|c|}
\hline Fig. No. & $\begin{array}{l}\text { Description of } \\
\text { experiment }\end{array}$ & $\begin{array}{c}\text { rms } \% \\
\text { deviation } \\
\text { (Maximum point } \\
\text { difference) }\end{array}$ & $\begin{array}{l}\text { DPM }(1 \sigma) \\
\text { uncertainty } \\
\text { range }(\%)\end{array}$ & $\begin{array}{l}\text { Estimated } \\
\text { total }(1 \sigma) \mathrm{MC} \\
\text { uncertainty } \\
\text { range }(\%)\end{array}$ \\
\hline $\begin{array}{l}4(\mathrm{a}) \\
\text { (upper) }\end{array}$ & $\begin{array}{l}6 \mathrm{MV} \text { photons, } 2 \times 2 \mathrm{~cm}^{2} \text {, } \\
\text { CAX depth dose in water }\end{array}$ & $\begin{array}{c}0.86 \\
(2.54)\end{array}$ & $0.39-0.78$ & $0.6-1.4$ \\
\hline $\begin{array}{l}\text { 4(a) } \\
\text { (middle) }\end{array}$ & $\begin{array}{l}6 \mathrm{MV} \text { photons, } 3 \times 3 \mathrm{~cm}^{2} \text {, } \\
\text { CAX depth dose in water }\end{array}$ & $\begin{array}{c}0.75 \\
(1.68)\end{array}$ & $0.36-0.74$ & $0.6-1.3$ \\
\hline $\begin{array}{l}4(\mathrm{a}) \\
\text { (lower) }\end{array}$ & $\begin{array}{l}6 \mathrm{MV} \text { photons, } 10 \times 10 \mathrm{~cm}^{2} \text {, } \\
\text { CAX depth dose in water }\end{array}$ & $\begin{array}{c}0.55 \\
(-1.53)\end{array}$ & $0.75-1.05$ & $1.0-1.6$ \\
\hline $\begin{array}{l}4(\mathrm{~b}) \\
\text { (upper) }\end{array}$ & $\begin{array}{l}15 \mathrm{MV} \text { photons, } 2 \times 2 \mathrm{~cm}^{2} \\
\text { CAX depth dose in water }\end{array}$ & $\begin{array}{r}0.77 \\
(-2.90)\end{array}$ & $0.07-0.14$ & $0.5-1.1$ \\
\hline $\begin{array}{l}4(\mathrm{~b}) \\
\text { (middle) }\end{array}$ & $\begin{array}{l}15 \mathrm{MV} \text { photons, } 3 \times 3 \mathrm{~cm}^{2} \text {, } \\
\text { CAX depth dose in water }\end{array}$ & $\begin{array}{c}0.87 \\
(-2.14)\end{array}$ & $0.16-0.32$ & $0.5-1.2$ \\
\hline $\begin{array}{l}4(\mathrm{c}) \\
\text { (lower) }\end{array}$ & $\begin{array}{l}15 \mathrm{MV} \text { photons, } 10 \times 10 \mathrm{~cm}^{2} \text {, } \\
\text { CAX depth dose in water }\end{array}$ & $\begin{array}{c}0.67 \\
(-2.64)\end{array}$ & $0.34-0.64$ & $0.7-1.4$ \\
\hline $\begin{array}{l}7 \text { (a) } \\
\text { (upper) }\end{array}$ & $\begin{array}{l}6 \mathrm{MV} \text { photons, } 2 \times 2 \mathrm{~cm}^{2} \text {, } \\
\text { CAX depth dose in } \\
\text { water/lung/water }\end{array}$ & $\begin{array}{c}0.81 \\
(-2.36)\end{array}$ & $0.08-0.16$ & $0.5-1.1$ \\
\hline $\begin{array}{l}7(\mathrm{a}) \\
\text { (middle) }\end{array}$ & $\begin{array}{l}6 \mathrm{MV} \text { photons, } 3 \times 3 \mathrm{~cm}^{2} \text {, } \\
\text { CAX depth dose in } \\
\text { water/lung/water }\end{array}$ & $\begin{array}{c}0.56 \\
(-1.12)\end{array}$ & $0.12-0.22$ & $0.5-1.1$ \\
\hline $\begin{array}{l}7(\mathrm{a}) \\
\text { (lower) }\end{array}$ & $\begin{array}{l}6 \mathrm{MV} \text { photons, } 10 \times 10 \mathrm{~cm}^{2} \text {, } \\
\text { CAX depth dose in } \\
\text { water/lung/water }\end{array}$ & $\begin{array}{c}0.72 \\
(-1.88)\end{array}$ & $0.34-0.60$ & $0.7-1.4$ \\
\hline $\begin{array}{l}7 \text { (b) } \\
\text { (upper) }\end{array}$ & $\begin{array}{l}15 \mathrm{MV} \text { photons, } 2 \times 2 \mathrm{~cm}^{2} \text {, } \\
\text { CAX depth dose in } \\
\text { water/lung/water }\end{array}$ & $\begin{array}{c}1.01 \\
(-2.64)\end{array}$ & $0.07-0.11$ & $0.5-1.1$ \\
\hline $\begin{array}{l}7(\mathrm{~b}) \\
\text { (middle) }\end{array}$ & $\begin{array}{l}15 \mathrm{MV} \text { photons, } 3 \times 3 \mathrm{~cm}^{2} \text {, } \\
\text { CAX depth dose in } \\
\text { water/lung/water }\end{array}$ & $\begin{array}{c}1.00 \\
(-2.65)\end{array}$ & $0.10-0.16$ & $0.5-1.1$ \\
\hline $\begin{array}{l}7(\mathrm{~b}) \\
\text { (lower) }\end{array}$ & $\begin{array}{l}15 \mathrm{MV} \text { photons, } 10 \times 10 \mathrm{~cm}^{2} \text {, } \\
\text { CAX depth dose in } \\
\text { water/lung/water }\end{array}$ & $\begin{array}{r}0.81 \\
(-1.0)\end{array}$ & $0.25-0.37$ & $0.7-1.3$ \\
\hline
\end{tabular}

manufacturer (Gammex, RMI, Middleton, WI). Measurements of the density of the lung-equivalent slabs, based on CT-scans and a physical measurement of the mass and volume, agreed with the value of $0.3 \mathrm{~g} / \mathrm{cm}^{3}$, as provided by the manufacturer. All DPM calculations were performed using a 1 $\mathrm{mm}$ step size, and low energy electron and photon cutoffs of $200 \mathrm{keV}$ and $50 \mathrm{keV}$, respectively.

DPM is implemented on a linux-based platform consisting of multiple, $1.4 \mathrm{GHz}$ (AMD Athlone) processors that are configured to process calculations in parallel. The shared cluster is owned and maintained by the University of Michigan Center for Advanced Computing. For this study, the number of processors for each calculation was dependent upon the availability of free processors, but typically ranged from 2 to 25 . The time to process one hundred million histories on a single processor was approximately $0.9 \mathrm{~h}$ and 1.2 $\mathrm{h}$ for the 6 and $15 \mathrm{MV}$ photon beams, respectively. The reduction in computing time was found to scale almost linearly with the number of processors with some minimal overhead associated with file $\mathrm{I} / \mathrm{O}$.

\section{SIMULATION RESULTS AND DISCUSSION}

\section{A. Homogeneous phantom benchmarks}

Figure 3 shows the central axis, normalized, bremsstrahlung spectra, differential in energy, for the 6 and $15 \mathrm{MV}$ photon beams. The spectra were reconstructed, using BEAMDP, from the "open beam" phase space scored at phase space plane I in Fig. 1. Electron-on-target energies were 6.25 and $15.3 \mathrm{MeV}$ for the 6 and $15 \mathrm{MV}$ photons, respectively. From these figures, the average photon energies were computed to be $1.7 \mathrm{MeV}(6 \mathrm{MV})$ and $3.62 \mathrm{MeV}(15 \mathrm{MV})$. Figures 4(a) and 4(b) illustrate the relative central axis doses as a function of depth in water for the 6 and $15 \mathrm{MV}$ photon beams respectively. DPM calculations are depicted with open markers and diode measurements are shown in the solid lines. Each figure depicts three sets of curves, corresponding to the square field sizes at the isocenter, $2 \times 2,3 \times 3$, and $10 \times 10 \mathrm{~cm}^{2}$, tested in this study. The curves have all been normalized at a depth of $10 \mathrm{~cm}$; the $2 \times 2$ and $3 \times 3 \mathrm{~cm}^{2}$ fields include additional scaling factors of 0.50 and 0.75 , respectively, for ease of illustration. The root-mean-square 


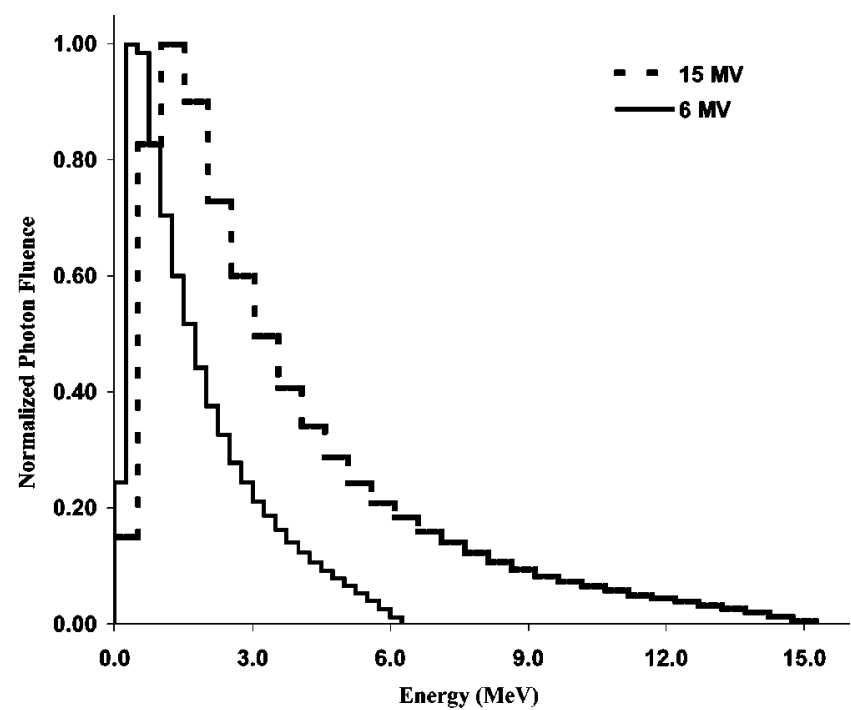

FIG. 3. Normalized photon fluence as a function of energy, along the central axis, for 6 and $15 \mathrm{MV}$ photon beams. These bremsstrahlung spectra were reconstructed from phase space simulation of the linear accelerator treatment head using BEAMnrc. The electron-on-target energies were $6.25 \mathrm{MeV}$ and $15.3 \mathrm{MeV}$ for the 6 and $15 \mathrm{MV}$ photon beams, respectively.

(rms) values of the percentage differences between calculations and measurements, as well as the maximum differences, are presented in Table I for all depth dose curves shown in Fig. 4. The percent differences were calculated using the relation: (calculated value-measured value) $\times 100$ / maximum measured point value. Also included in Table I are the DPM $(1 \sigma)$ uncertainties for the in-phantom calculations, as well as the total Monte Carlo uncertainty, at the $1 \sigma$ level. The total uncertainty was evaluated as a quadrature sum of the approximate latent uncertainty in the phase space file and the DPM uncertainty, due only to random fluctuations in phantom, as explained in Sec. IV A. As seen in Table I, DPM calculations are in within $1 \%$ agreement with measurements (based on rms \% deviation) for all square field depth dose curves presented in Figs. 4(a) and 4(b). The rms \% deviations are also within the estimated total Monte Carlo uncertainty ranges provided in Table I. Despite the good average agreement, we find maximum point differences in the range from $-1.5 \%$ to $-3 \%$ for the square field depth dose comparisons. The maximum point differences were all found to occur in the buildup region, where we estimate the experimental uncertainty to be on the order of $0.5 \mathrm{~mm}$, or $5-10 \%$ per $\mathrm{mm}$. Therefore, we attribute some part of these differences to the setup uncertainty. However, we also note in Table I that the maximum point differences for the $15 \mathrm{MV}$ beam for the depth dose curves in Fig. 4(b) were all negative, indicating that the DPM calculated dose is less than measurements in the buildup region. This may lead one to suspect that there is potentially a systematic error that has not been accounted for. In fact, this may indeed be the case. One of the limitations of this study is that we have not thoroughly investigated buildup dose issues. It is well known that accurate measurement of dose (within $\pm 3 \%$ uncertainty) requires an accurate setup as well as the use of extrapolation or par-
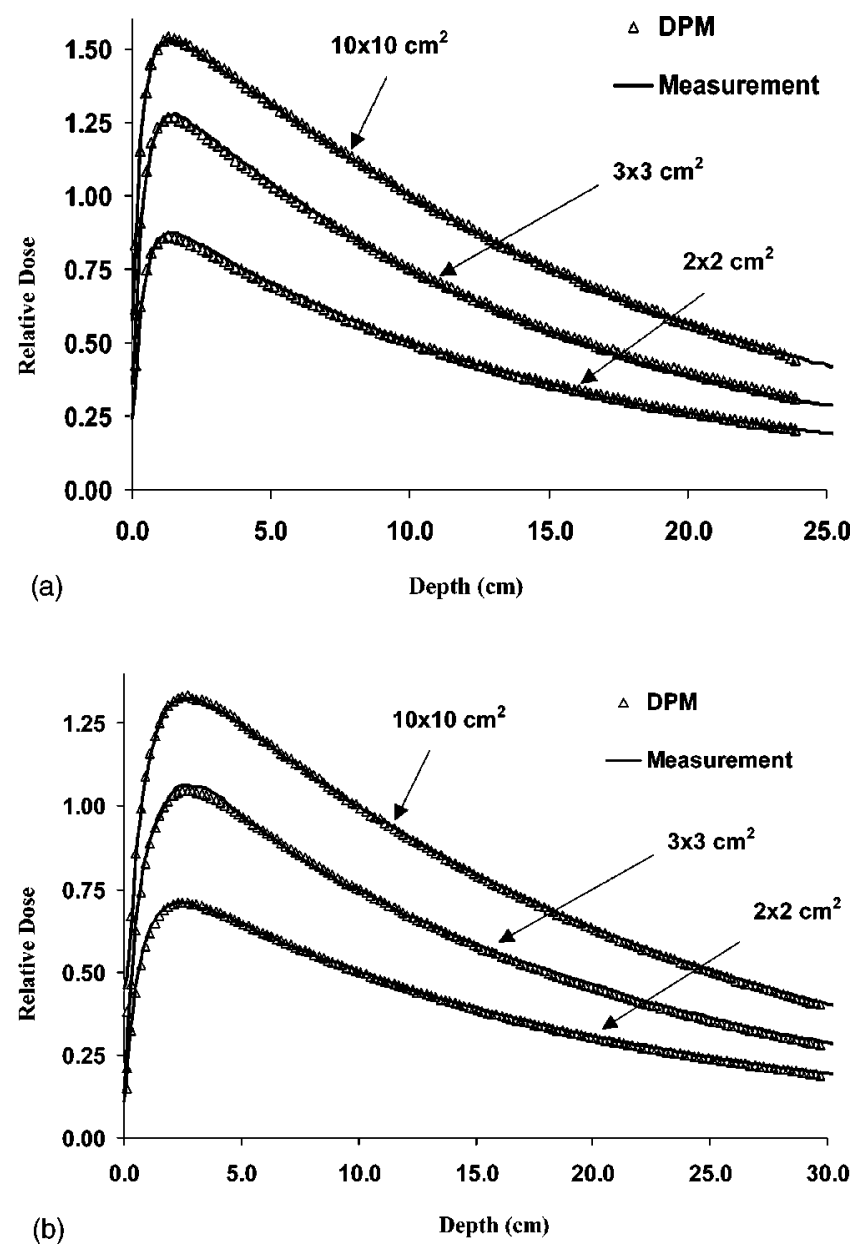

FIG. 4. Relative central axis depth dose for (a) $6 \mathrm{MV}$ and (b) $15 \mathrm{MV}$ photons in a water phantom. DPM calculations are shown with markers and diode measurements are shown in the solid lines. Curves are illustrated for field sizes of $2 \times 2,3 \times 3$, and $10 \times 10 \mathrm{~cm}^{2}$. Depth dose curves have been normalized to the point dose at a depth of $10 \mathrm{~cm}$ for the respective field size. The $2 \times 2$ and $3 \times 3 \mathrm{~cm}^{2}$ curves have been scaled using factors of 0.5 and 0.75 , respectively, for an illustration on the same graph.

allel plane chambers. However, it is equally likely that the Monte Carlo calculations do not accurately (within $\pm 3 \%$ ) predict the dose in the buildup region. ${ }^{7,30}$ For example, Hartmann Siantar et al. $^{7}$ have shown that their source calculations, conducted with PEREGRINE and based on phase space calculations from BEAM, required additional electrons to agree with measurements in the buildup region for field sizes ranging from $2 \times 2-38 \times 38 \mathrm{~cm}^{2}$. Although the effect of contaminant electrons at small field sizes is small, it is nevertheless a potential source of the point dose disagreements observed in the buildup region. We are currently conducting a study of accurate dose measurements in the buildup region; ${ }^{31}$ this data will be used to benchmark Monte Carlo calculated buildup doses over a range of clinically relevant field sizes.

Figures 5(a), 5(b), and 5(c) illustrate the $6 \mathrm{MV}$ central axis profile doses along the $x$-axis in water for the $2 \times 2$, $3 \times 3$, and $10 \times 10 \mathrm{~cm}^{2}$ fields, respectively. DPM calculations are shown in open markers and diode measurements are shown in solid lines. Each figure depicts three curves, corresponding to profiles at depths of $1.5 \mathrm{~cm}\left(d_{\max }\right), 10 \mathrm{~cm}$, and 

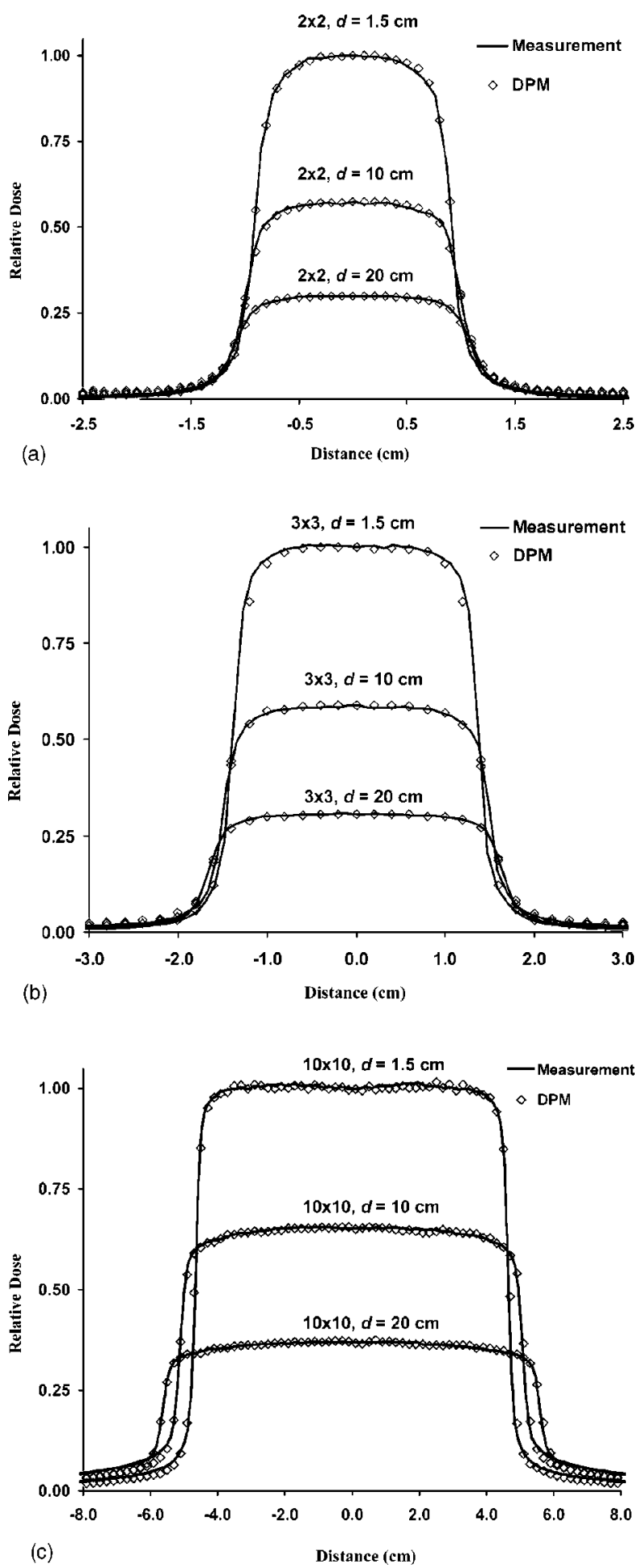

FIG. 5. Relative central axis profile doses for $6 \mathrm{MV}$ photons in a water phantom. DPM calculations are shown with markers and diode measurements are shown in the solid lines. Profiles are illustrated for (a) $2 \times 2 \mathrm{~cm}^{2}$, (b) 3 $\times 3 \mathrm{~cm}^{2}$, and (c) $10 \times 10 \mathrm{~cm}^{2}$ field sizes, at depths of $d_{\max }(1.5 \mathrm{~cm}), 10$, and $20 \mathrm{~cm}$. All curves have been normalized to the maximum central axis point dose.

$20 \mathrm{~cm}$. All curves have been normalized to the maximum point dose along the central axis. The $1 \sigma$ total Monte Carlo uncertainties in these calculations ranged from less than $1 \%$ for the $2 \times 2$, and $3 \times 3 \mathrm{~cm}^{2}$ fields to less than $2 \%$ for the 10 $\times 10 \mathrm{~cm}^{2}$ field size. Analyses of the differences between calculations and measurements for the profiles illustrated in Fig. 5 reveals that the average agreement is within (a) $1 \%$ in the inner beam region (dose $>90 \%$ ), (b) $1 \mathrm{~mm}$ distance-toagreement in the penumbral region $(20 \%<$ dose $<80 \%)$, and (c) $2 \%$ in the outer beam region (dose $<20 \%$ ). These differences are within the acceptability criteria specified by the AAPM Task Group No. $53^{32}$ for profile doses in homogeneous media. These suggested criteria are $2 \%$ (of the maximum central axis dose) in the inner beam region, $2 \mathrm{~mm}$ distance-to-agreement in the profile penumbral region, and $2 \%$ (of the maximum central axis dose) in the outer beam region. ${ }^{32}$ Relative profile doses for the $2 \times 2,3 \times 3$, and 10 $\times 10 \mathrm{~cm}^{2}, 15 \mathrm{MV}$ photon fields are presented in Figs. 6(a), 6(b), and 6(c), respectively. Profiles at each field size were acquired at depths of $3.0 \mathrm{~cm}\left(d_{\max }\right), 10 \mathrm{~cm}$ and $20 \mathrm{~cm}$. As with the $6 \mathrm{MV}$ profiles, all curves have been normalized to the maximum central axis point dose. The Monte Carlo uncertainties associated with these curves are similar to those found for the $6 \mathrm{MV}$ profile calculations. The differences versus measurements for the $15 \mathrm{MV}, 2 \times 2 \mathrm{~cm}^{2}$ [Fig. 6(a)] and $3 \times 3 \mathrm{~cm}^{2}$ [Fig. 6(b)] DPM profiles are comparable to those for the corresponding $6 \mathrm{MV}$ profiles, with the exception of the $10 \times 10 \mathrm{~cm}^{2}$ [Fig. 6(c)], where the $15 \mathrm{MV}$ calculated profile penumbrae show a greater difference from measurements relative to the $6 \mathrm{MV}$ beam [Fig. 5(c)]. This discrepancy is attributed to a slight misalignment in the jaw positions for the $15 \mathrm{MV}$ beam. As reported by Bagheri et al., ${ }^{33}$ an uncertainty of $0.05 \mathrm{~cm}$ in the lateral jaw position (located at $z$ $=39 \mathrm{~cm}$ in their study) can cause changes of up to $8 \%$ of the maximum dose in the penumbral region. Given that the measurements and calculations in this work were conducted with an estimated uncertainty of $0.1 \mathrm{~cm}(2 \sigma)$ in the jaw positions, the penumbral differences are found to be within the experimental uncertainty.

\section{B. Inhomogeneous phantom benchmarks}

Figures 7(a) and 7(b) illustrate the central axis depth dose comparisons for 6 and $15 \mathrm{MV}$ photons, respectively, within the inhomogeneous (composite solid water/lung/solid water) phantom. Depth doses for the $2 \times 2,3 \times 3$, and $10 \times 10 \mathrm{~cm}^{2}$ (specified at the isocenter) have all been plotted on the same graph. DPM calculations are shown in the solid lines with ion chamber measurements depicted in open markers. All curves have been normalized to the doses for the respective energies and field sizes at a depth of $10 \mathrm{~cm}$ in the homogeneous (water only) situation. The $2 \times 2$ and $3 \times 3 \mathrm{~cm}^{2}$ field sizes include scaling factors of 0.5 and 0.75 , respectively, so that all curves can be viewed on the same plot. The rms deviations of the differences between calculations and measurements as well as the Monte Carlo uncertainties, at the $1 \sigma$ level, are presented in Table I for the depth dose curves in Figs. 7(a) and 7(b). The rms deviations for both energies and all field sizes are within $1 \%$, and fall within the estimated Monte Carlo uncertainty ranges. Maximum point differences, from $-1 \%$ to $-2.7 \%$, are evident, however, these are deemed to be within the $1 \mathrm{~mm}$ experimental uncertainty with 


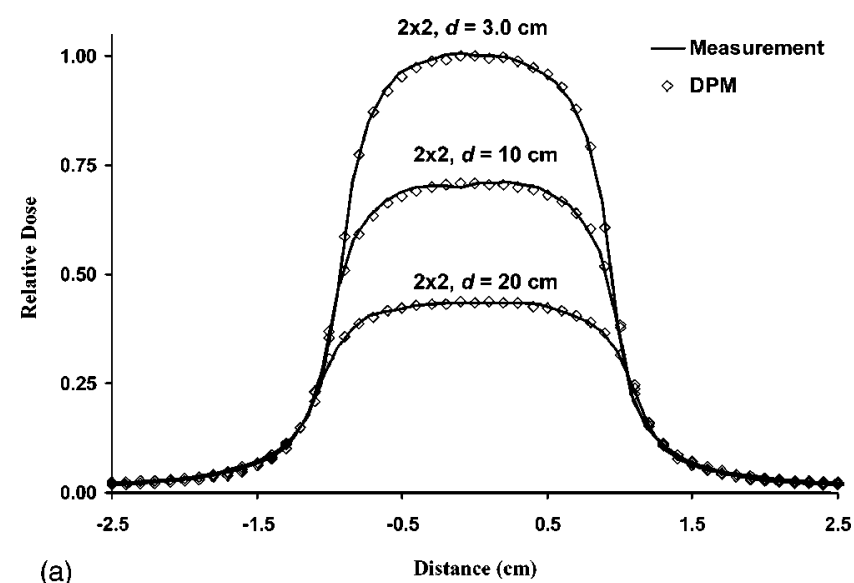

(a)

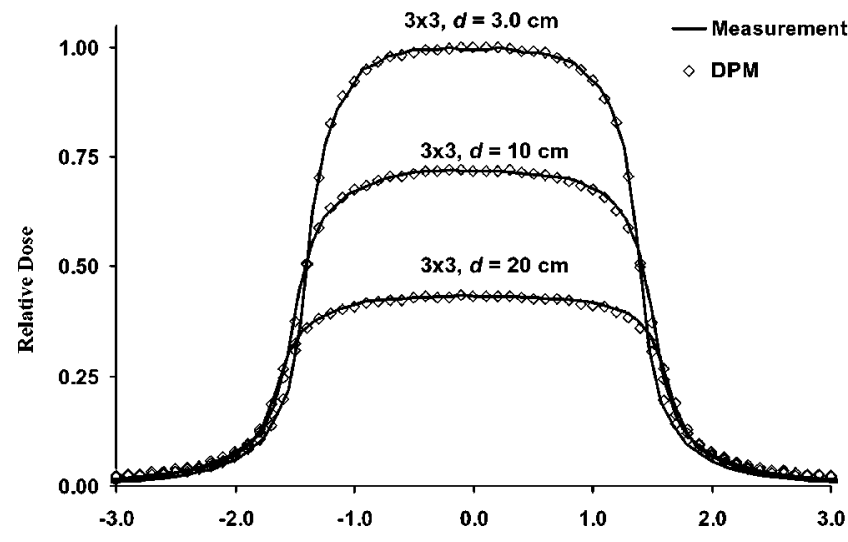

(b)

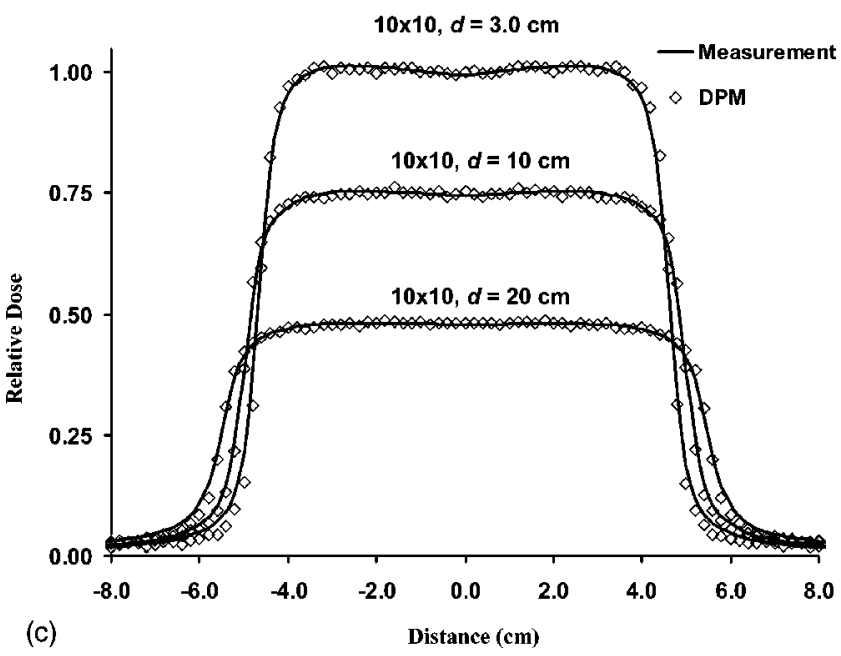

FIG. 6. Relative central axis profile doses for $15 \mathrm{MV}$ photons in a water phantom. DPM calculations are shown with markers and diode measurements are shown in the solid lines. Profiles are illustrated for (a) $2 \times 2 \mathrm{~cm}^{2}$, (b) 3 $\times 3 \mathrm{~cm}^{2}$, and (c) $10 \times 10 \mathrm{~cm}^{2}$ field sizes at depths of $d_{\max }(3.0 \mathrm{~cm}), 10$, and $20 \mathrm{~cm}$. All curves have been normalized to the maximum central axis point dose.

respect to depth positioning of the ion chamber. The AAPM Task Group No. $53^{32}$ suggested that acceptability criteria for slab inhomogeneities along the central axis is 3\%; this excludes regions of electronic disequilibrium and is therefore
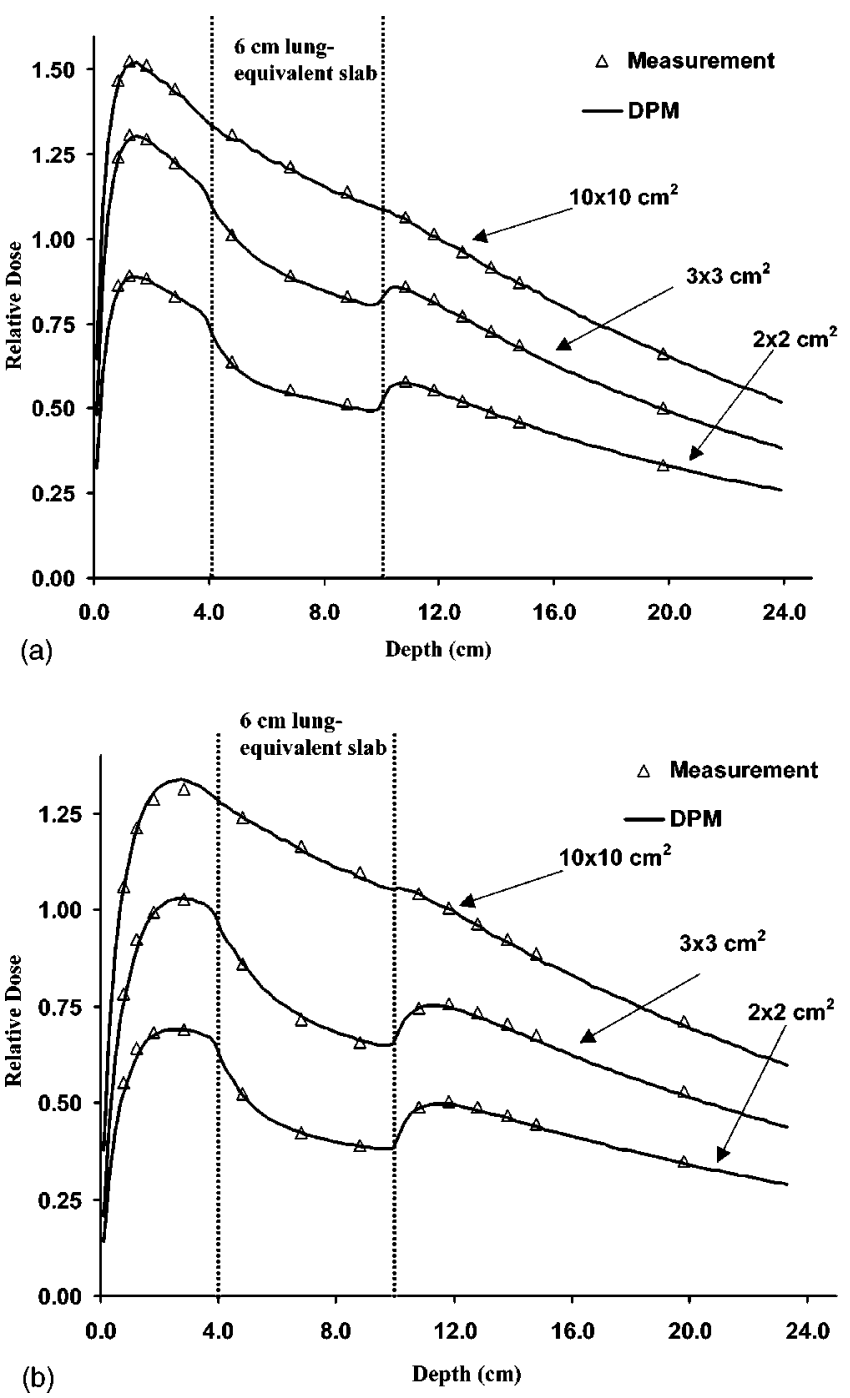

FIG. 7. Relative central axis depth dose for (a) $6 \mathrm{MV}$ and (b) $15 \mathrm{MV}$ photons in the inhomogeneous (solid-water/lung/solid-water) phantom. DPM calculations are shown in the solid lines and ion chamber measurements are shown with markers. Curves are illustrated for field sizes of $2 \times 2,3 \times 3$, and 10 $\times 10 \mathrm{~cm}^{2}$. Depth dose curves have been normalized to the doses, for the respective field sizes, at $10 \mathrm{~cm}$ depth in the homogeneous phantom. The 2 $\times 2$ and $3 \times 3 \mathrm{~cm}^{2}$ curves have been scaled using factors of 0.5 and 0.75 , respectively, for an illustration on the same graph.

only applicable in our study for the largest field size (10 $\times 10 \mathrm{~cm}^{2}$ ). It is clear that more suitable difference criteria are necessary for experiments involving regions of electronic disequilibrium.

Several important physical phenomena are observable in the depth dose curves within the inhomogeneous phantom presented in Fig. 7. We note a severe reduction in dose within the lung-equivalent slab, which tends to worsen as the field size is reduced. This is due to the loss of lateral electronic equilibrium at the smaller field sizes $(2 \times 2$ and 3 $\times 3 \mathrm{~cm}^{2}$ ), coupled with a reduction of photon scatter in the low-density medium. The loss of lateral electronic equilibrium is greater for higher energy photons because of the increased range of the lateral electrons; this is exemplified for the $15 \mathrm{MV}$ photon beam in Fig. 7(b) where the reduction 
in dose within the inhomogeneity at smaller field sizes is greater than that of the $6 \mathrm{MV}$ beam [Fig. 7(a)]. As the field size is increased the reduction in dose within the lung becomes less pronounced, and for a $10 \times 10 \mathrm{~cm}^{2}$ field, the effect of the inhomogeneity is much less discernible. At depths immediately beyond the distal end of the inhomogeneity, the dose increases because of the reduced attenuation in the lung slab as well as the increase in electron backscatter from the higher-density water. Similar results have been reported by other investigators, ${ }^{34,35}$ albeit for larger field sizes. It is instructive to present the depth dose curves in Fig. 7 in the form of lung dose correction factors $(\mathrm{CF})$, where the $\mathrm{CF}$ is defined at a given depth on the central axis as the dose in the heterogeneous phantom divided by the dose in a solid water phantom. The CFs provide a more quantitative means of evaluating the influence of the inhomogeneity on the depth dose. CFs are illustrated as a function of depth and field size in Figs. 8(a) and 8(b) for 6 and 15 MV photons, respectively. DPM calculated CFs are shown as dashed lines with open markers, while those for the measurements are shown with solid markers. Differences between calculated and measured CFs are similar to those observed for the depth dose comparisons in Fig. 7. As seen with the depth dose curves in Fig. 7, there is a reduction in CFs (in Fig. 8) as the field size is reduced. The dose reduction is greater at $15 \mathrm{MV}$; for example, the lowest CF for a $2 \times 2 \mathrm{~cm}^{2}$ field size is approximately 0.85 for $6 \mathrm{MV}$ [Fig. 8(a)], while it is 0.70 for $15 \mathrm{MV}$ [Fig. 8(b)]; this follows from the fact that there is increased lateral electronic disequilibrium at $15 \mathrm{MV}$, as discussed earlier. Beyond the distal end of the interface the CFs increase above 1.0, indicating that the dose is higher in the solid water beyond the interface relative to that in the homogeneous situation. In addition, the CFs are higher at the smaller field sizes. These effects are attributed to the following factors: (a) reduced attenuation within the lung slab resulting in an increase in photon fluence in solid water beyond the lung relative to the homogeneous depth dose, and (b) more dose being transported away from the lung into the adjacent distal solid water at smaller field sizes, due to increased lateral electron transport issues. The correction factors in solid water beyond the lung are also found to be higher at $6 \mathrm{MV}$ relative to 15 MV. This is due to the reduction in photon attenuation which results when water is replaced with lung in the composite phantom. As the average attenuation coefficient is higher at 6 MV than at $15 \mathrm{MV}$, this effect (and hence the CFs) will be larger at $6 \mathrm{MV}$ in the water medium beyond the lungequivalent slab. The CFs reported in this study are in good agreement with those measured by Rice et al. ${ }^{34}$ for 4 and 15 MV photon beams.

Figures 9(a) and 9(b) show the relative profile dose comparisons in the inhomogeneous phantom for 6 and $15 \mathrm{MV}$ photons, respectively. Each figure illustrates profiles for the $2 \times 2$ and $10 \times 10 \mathrm{~cm}^{2}$ field sizes at depths of $8 \mathrm{~cm}$ (within the lung slab) and $12 \mathrm{~cm}$ (in the solid water beyond the lung). All profiles have been normalized to the respective central axis doses; the profiles at a depth of $12 \mathrm{~cm}$ contain an additional scaling factor of 0.75 for representation on the same graph. DPM calculations are depicted with open mark-
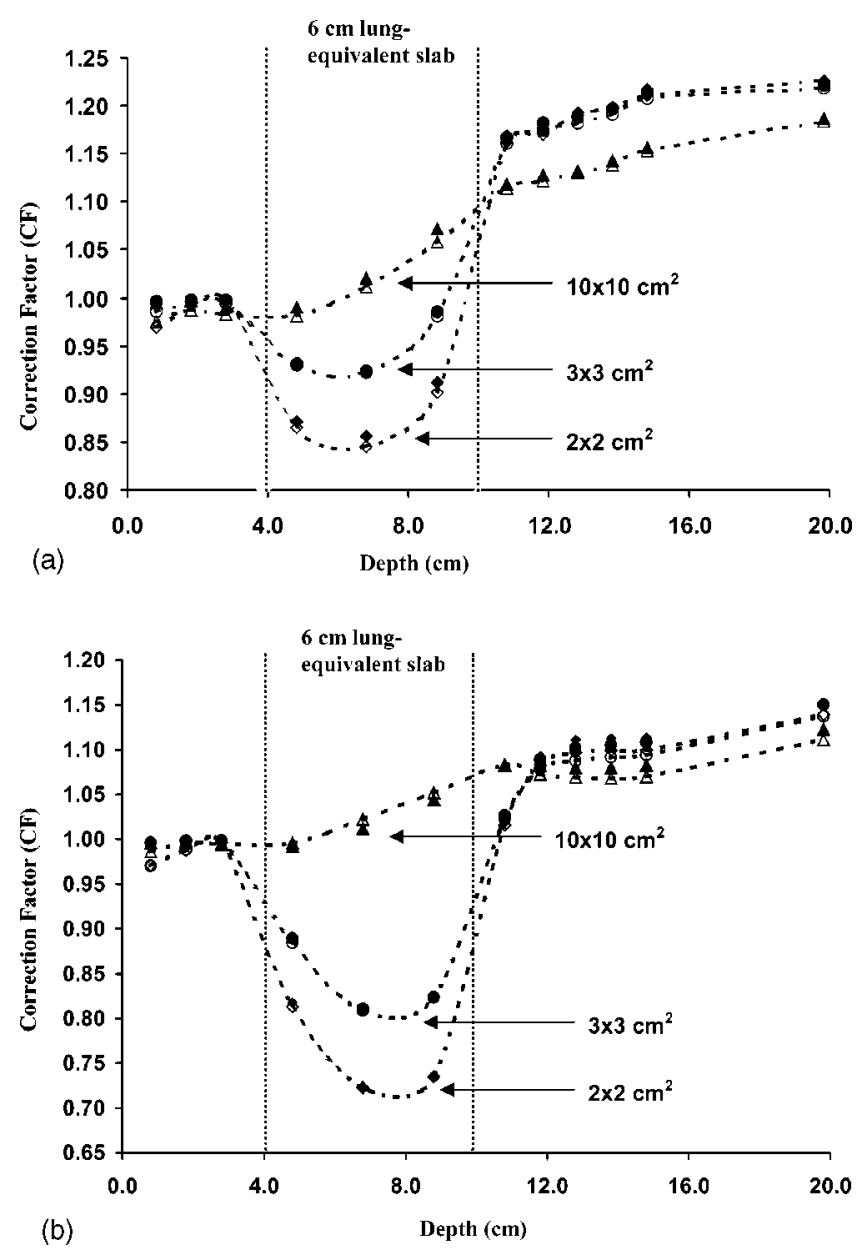

FIG. 8. Correction factors $(\mathrm{CF})$ as a function of depth for the depth dose curves illustrated in Fig. 7, for (a) $6 \mathrm{MV}$ and (b) $15 \mathrm{MV}$ photons. The CF is defined as the ratio of dose in the inhomogeneous phantom to that in the homogeneous (water) phantom, at a given field size and depth. DPM calculated CFs are shown as solid lines with open markers and measured CFs are shown with closed markers. CFs are illustrated for field sizes of $2 \times 2,3 \times 3$, and $10 \times 10 \mathrm{~cm}^{2}$.

ers, ion chamber measurements with closed markers and EDR film measurements with solid lines. The $1 \sigma$ Monte Carlo uncertainties were, on average, less than $1 \%$ for the $2 \times 2 \mathrm{~cm}^{2}$ field and less than $2 \%$ for $10 \times 10 \mathrm{~cm}^{2}$ field size. The differences between DPM calculations and measurements are generally within (a) $2 \%$ in the inner beam region (dose $>90 \%$ ), (b) $1 \mathrm{~mm}$ distance-to-agreement in the penumbral region $(20 \%<$ dose $<80 \%)$ when compared with EDR film measurements, and (c) $2 \%$ in the outer beam region (dose $<20 \%$ ). The lateral broadening of the profile penumbral regions within the lung slab is also illustrated in Fig. 9. The profile penumbral regions are consistently broader at a depth of $8 \mathrm{~cm}$ due to the lateral spreading of dose within the lung; this effect is more significant for the $2 \times 2 \mathrm{~cm}^{2}$ profiles. An estimate of the $80 \%-20 \%$ penumbral widths for the 2 $\times 2 \mathrm{~cm}^{2}$ field at depths of $8 \mathrm{~cm}$ and $12 \mathrm{~cm}$-including an inverse-square correction so that both profiles are at the same distance from the source-gives values of $4.6 \mathrm{~mm}(d$ $=8 \mathrm{~cm})$ and $3 \mathrm{~mm}(d=12 \mathrm{~cm})$, for the $6 \mathrm{MV}$ beam. The corresponding penumbra values for the $15 \mathrm{MV}$ beam (2 


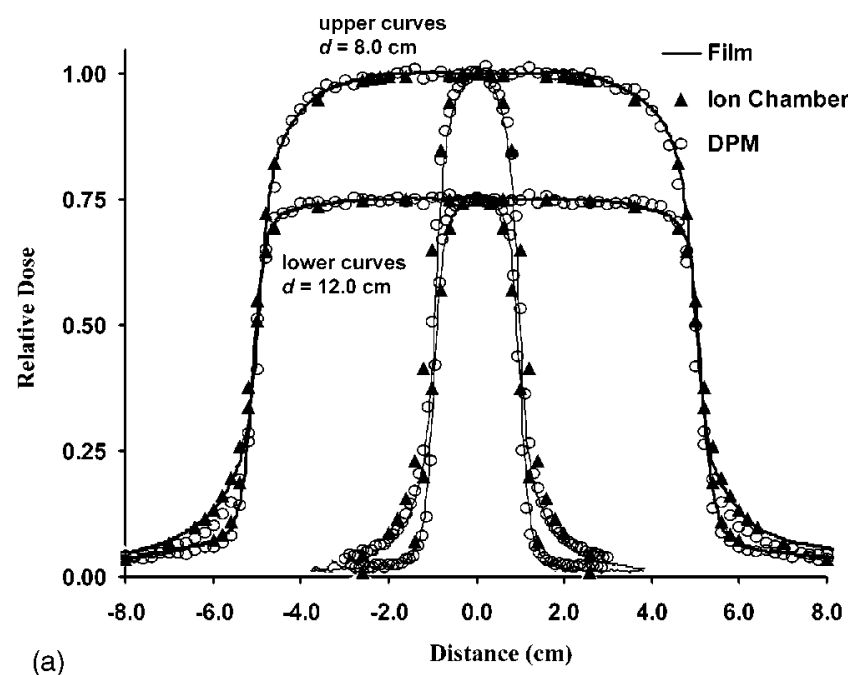

(a)

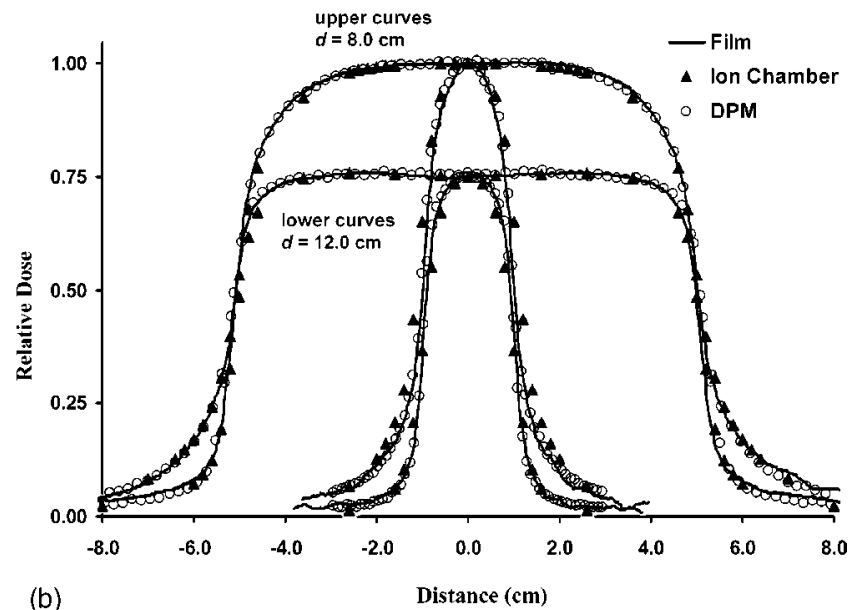

FIG. 9. Relative central axis profile doses for (a) $6 \mathrm{MV}$ and (b) $15 \mathrm{MV}$ photons in the inhomogeneous (solid-water/lung/solid-water) phantom. DPM calculations are shown with open markers, ion chamber measurements with closed markers, and film measurements with solid lines. Profiles are illustrated for field sizes of $2 \times 2$ and $10 \times 10 \mathrm{~cm}^{2}$ at depths of $8 \mathrm{~cm}$ (within the lung) and $12 \mathrm{~cm}$ (within the solid water). Curves have been normalized to their respective central axis doses; profiles at a depth of $12 \mathrm{~cm}$ include a scaling factor of 0.75 for illustration purposes.

$\times 2 \mathrm{~cm}^{2}$ field $)$ are $6.0 \mathrm{~mm}(d=8 \mathrm{~cm})$ and $4.2 \mathrm{~mm}(d$ $=12 \mathrm{~cm}$ ). Finally, it is observed that the profile penumbral broadening for the $2 \times 2 \mathrm{~cm}^{2}$ field within the lung is largest with the ion chamber measurements. This is due to the volume averaging effect of the ion chamber, which appears to be most prominent on the small field profiles in the lung, where the lateral scattering of electrons is significant. Given that the ion chamber has a $3 \mathrm{~mm}$ inner radius, it is not unexpected that the penumbral blurring (due to volume averaging) is most pronounced for the $2 \times 2 \mathrm{~cm}^{2}$ profiles.

\section{CONCLUSION}

Our goal in this investigation was to benchmark the accuracy of the DPM Monte Carlo code for photon dose calculations in a low-density, lung-equivalent medium, using small field sizes, where lateral electronic disequilibrium effects are maximized. In order to properly evaluate the radiation trans- port accuracy of the DPM code under the aforementioned conditions, it was necessary to first benchmark the accuracy of the phase-space calculations of the linear accelerator treatment (conducted with BEAMnrc) in a homogeneous water phantom for field sizes of $2 \times 2,3 \times 3$, and $10 \times 10 \mathrm{~cm}^{2}$ (specified at the isocenter). DPM central axis depth dose and profile calculations for $2 \times 2,3 \times 3$, and $10 \times 10 \mathrm{~cm}^{2}$ field sizes in water were, on average, within $\pm 1 \% / 1 \mathrm{~mm}$ relative agreement with diode measurements. The homogeneous phantom study illustrated that the phase space simulations provided an accurate description of the radiation source, the intent being that any differences noted in the inhomogeneous situation would not be due to issues regarding the radiation source. The inhomogeneous phantom experiment, conducted in a solid-water/lung/solid-water phantom, involved the use of an ion chamber and film for depth dose and profile measurements at depths within and beyond the lung. DPM calculations were generally within $\pm 2 \%$ relative agreement with measurements for all depth dose and profile comparisons (in the inner and outer beam regions) and within 1-2 $\mathrm{mm}$ distance-to-agreement in the profile penumbral regions, for all field sizes in the inhomogeneous phantom.

Although this work has demonstrated that the DPM Monte Carlo code is capable, even under conditions of severe electronic disequilibrium, of accurate photon beam dose calculations in lung-equivalent media, we realize that much more testing and validation is necessary before DPM is clinically useful. For example, the issue of accurate dose calculations and measurements in the depth dose buildup region is important and requires further investigation. This is an issue that is quite clinically relevant, especially in anatomical regions, such as the head and neck, where lesions can be superficially seated. Another important topic involves the phase space simulation of the accelerator treatment head. Sheikh-Bagheri and Rogers ${ }^{36}$ have recently shown that accelerator head modeling is sensitive to issues such as the incident electron-ontarget energy spectrum and angular distribution. Although we have found the use of mono-energetic, mono-directional electrons-on-target to be an accurate representation of the fields sizes studied in this work, it is possible that this approximation breaks down for larger field sizes (greater than $10 \times 10 \mathrm{~cm}^{2}$ ). These issues along with others will be the focus of future work.

\section{ACKNOWLEDGMENTS}

This work has been supported in part by NIH Grant No. P01-CA59827 and by a University of Michigan Cancer Center grant funded by the John and Suzanne Munn Endowment. We are indebted to Dave Rogers, and the BEAM group at the NRCC, Blake Walters, in particular, for his assistance with all our questions. We are grateful to Dr. Bose of the UM Center for Advanced Computing, for his assistance with parallelizing DPM on the shared linux cluster. We would like to thank Gary Engeseth of Gammex, RMI (Gammex, RMI, Middleton, WI) for providing the atomic composition of the lung-equivalent material, and Varian Medical Systems 
(Varian Associates, Palo Alto, CA) for providing proprietary information on the design of the linear accelerator.

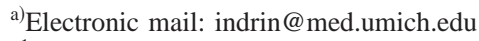

${ }^{1}$ J. Van Dyke, R. B. Barnett, J. E. Cygler, and P. C. Shragge, "Commissioning and quality assurance of treatment planning computers," Int. J. Radiat. Oncol., Biol., Phys. 26, 261-273 (1992).

${ }^{2}$ P. Andreo, "Monte Carlo techniques in medical radiation physics," Phys. Med. Biol. 26, 861-920 (1991).

${ }^{3}$ T. R. Mackie, J. W. Scrimger, and J. J. Battista, "A convolution method of calculating dose for 15 MV X-rays," Med. Phys. 12, 188-196 (1985).

${ }^{4}$ R. Mohan, "Why Monte Carlo?," in Proceedings of the XIIth International Conference on the Use of Computers in Radiation Therapy, Salt Lake City, UT, edited by D. D. Leavitt and G. Starkschall (Medical Physics Publishing, Madison, WI, 1997), pp. 16-18.

${ }^{5}$ M. R. Arnfield, C. Hartmann Siantar, J. Siebers, P. Garmon, L. Cox, and R. Mohan, "The impact of electron transport on the accuracy of computed dose," Med. Phys. 27, 1266-1274 (2000).

${ }^{6}$ A. E. Schach von Wittenau, L. J. Cox, P. M. Bergstrom, W. P. Chandler, and C. L. Hartmann-Siantar, "Correlated histogram representation of Monte Carlo derived medical accelerator photon output phase space," Med. Phys. 26, 1196-1212 (2000).

${ }^{7}$ C. L. Hartmann Siantar et al., "Description and dosimetric verification of the PEREGRINE Monte Carlo dose calculation system for photon beams incident on a water phantom," Med. Phys. 28, 1322-1337 (2001).

${ }^{8}$ J. V. Siebers, P. J. Keall, J. O. Kim, and R. Mohan, "Performance benchmarks of the MCV Monte Carlo system," in Proceedings of the XIIIthInternational Conference on the Use of Computers in Radiation Therapy, edited by W. Schlegel and T. Bortfeld (Springer-Verlag, Berlin, Germany, 2000), pp. 129-131.

${ }^{9}$ L. Wang, C. S. Chui, and M. Lovelock, "A patient-specific Monte Carlo dose-calculation method for photon beams," Med. Phys. 25, 867-878 (1998).

${ }^{10}$ J. Sempau, S. J. Wilderman, and A. F. Bielajew, "DPM, a fast, accurate Monte Carlo code optimized for photon and electron radiotherapy treatment planning dose calculations," Phys. Med. Biol. 45, 2263-2291 (2000).

${ }^{11}$ I. J. Chetty, J. M. Moran, T. S. Nurushev, D. L. McShan, B. A. Fraass, S. J. Wilderman, and A. F. Bielajew, "Experimental validation of the DPM Monte Carlo code using minimally scattered electron beams in heterogeneous media," Phys. Med. Biol. 47, 1837-1851 (2002).

${ }^{12}$ I. Kawrakow, M. Fippel, and K. Friedrich, "3D electron dose calculation using a Voxel based Monte Carlo algorithm (VMC)," Med. Phys. 23, 445-457 (1996).

${ }^{13}$ M. Fippel, "Fast Monte Carlo dose calculation for photon beams based on the VMC electron algorithm," Med. Phys. 26, 1466-1475 (1999).

${ }^{14}$ J. Deng, S. B. Jiang, J. Li, T. Pawlicki, and C. M. Ma, "Photon beam characterization and modeling for Monte Carlo treatment planning," Phys. Med. Biol. 45, 411-427 (2000).

${ }^{15}$ C. M. Ma et al., "Clinical implementation of a Monte Carlo treatment planning system," Med. Phys. 26, 2133-2143 (1999).

${ }^{16}$ C. M. Ma, P. Reckwerdt, M. Holmes, D. W. O. Rogers, and B. Geiser, “DOSXYZ Users Manual," NRCC Report PIRS 509B(rev C), 1998.

${ }^{17}$ J. J. DeMarco, T. D. Solberg, and J. B. Smathers, "A CT-based Monte Carlo dosimetry tool for radiotherapy treatment planning and analysis," Med. Phys. 25, 1-11 (1998).

${ }^{18}$ M. K. Fix, M. Stampanoni, P. Manser, E. J. Born, R. Mini, and P. Rue- gsegger, "A multiple source model for $6 \mathrm{MV}$ photon beam dose calculations," Phys. Med. Biol. 46, 1407-1428 (2001).

${ }^{19}$ P. J. Keall and P. W. Hoban, "Super-Monte Carlo: A 3D electron beam dose calculation algorithm," Med. Phys. 23, 2023-2034 (1996).

${ }^{20}$ H. Neuenschwander, T. R. Mackie, and P. J. Reckwerdt, "MMC-a highperformance Monte Carlo code for electron beam treatment planning," Phys. Med. Biol. 40, 543-574 (1995).

${ }^{21}$ J. Sempau, A. Sánchez-Reyes, F. Salvat, H. Oulad ben Tahar, S. B. Jiang, and T. R. Mackie, "Monte Carlo simulation of electron beams from an accelerator head using PENELOPE," Phys. Med. Biol. 46, 1163-1186 (2001).

${ }^{22}$ J. M. Fernández-Varea, R. Mayol, J. Baró, and F. Salvat, "On the theory and simulation of multiple elastic scattering of electrons," Nucl. Instrum. Methods Phys. Res. B 73, 447-473 (1993).

${ }^{23}$ D. W. O. Rogers, D. A. Faddegon, G. X. Ding, C. M. Ma, J. We, and T. R. Mackie, "BEAM: A Monte Carlo code to simulate radiotherapy treatment units," Med. Phys. 22, 503-524 (1995).

${ }^{24}$ I. Kawrakow, "Accurate condensed history Monte Carlo simulation of electron transport. I. EGSnrc, the new EGS4 version," Med. Phys. 27, 485498 (2000).

${ }^{25}$ D. M. J. Lovelock, C. S. Chui, and R. Mohan, "A Monte Carlo model of photon beams used in radiation therapy," Med. Phys. 22, 1387-1394 (1995).

${ }^{26}$ I. J. Chetty and P. M. Charland, "Investigation of Kodak extended dose range (EDR) film for megavoltage photon beam dosimetry," Phys. Med. Biol. 47, 3629-3641 (2002)

${ }^{27}$ J. F. Briesmeister, "MCNPTM — A general Monte Carlo $N$ Particle transport code," Los Alamos National Laboratory Report LA-12625-M, 1997.

${ }^{28}$ B. R. B. Walters, I. Kawrakow, and D. W. O. Rogers, "History by history statistical estimators in the BEAM code system," Med. Phys. 29, 2745$2752(2002)$

${ }^{29}$ C. S. Reft, "Output calibration in solid water for high energy photon beams," Med. Phys. 16, 299-301 (1988).

${ }^{30}$ G. X. Ding, "Dose discrepancies between Monte Carlo calculations and measurements in the buildup region for a high-energy photon beam," Med. Phys. 29, 2459-2463 (2002).

${ }^{31}$ S. Yokoyama, P. Roberson, J. Moran, D. Litzenberg, and B. Fraass, "Buildup region dependence on photon dose delivery technique for IMRT," Med. Phys. 29, 1315 (2002) (abstract).

${ }^{32}$ B. Fraass, K. Doppke, M. Hunt, G. Kutcher, G. Starkschall, R. Stern, and J. Van Dyke, "American Association of Physicists in Medicine Radiation Therapy Committee Task Group 53: Quality assurance for clinical radiotherapy treatment planning," Med. Phys. 25, 1773-1829 (1998).

${ }^{33}$ D. Sheikh-Bagheri et al., "Comparison of measured and Monte Carlo calculated dose distributions from the NRC linac," Med. Phys. 27, 22562266 (2000).

${ }^{34}$ R. Rice, J. L. Hansen, L. M. Chin, B. J. Mijnheer, and B. E. Bjärngard, "The influence of ionization chamber and phantom design on the measurement of lung dose in photon beams," Med. Phys. 15, 884-890 (1988).

${ }^{35}$ E. El-Khatib, M. Evans, M. Pla, and J. R. Cunningham, "Evaluation of lung dose correction methods for photon irradiations of thorax phantoms," Int. J. Radiat. Oncol., Biol., Phys. 17, 871-878 (1989).

${ }^{36}$ D. Sheikh-Bagheri and D. W. O. Rogers, "Sensitivity of megavoltage photon beam Monte Carlo simulations to electron beam and other parameters," Med. Phys. 29, 379-390 (2002). 